\section{UMBRAL Y EL JUEGO DE LOS ALEJANDRINOS (I)}

\author{
María del Pilar Couceiro \\ Universidad Complutense \\ athens-katharos@telefonica.net
}

\section{UMBRAL AND THE PLAY ON 14-SYLLABLE VERSES (I)}

Cómo citar este artículo/Citation: Couceiro, M. P. (2015). “Umbral y el juego de los alejandrinos (I)". Arbor, 191 (774): a248. doi: http://dx.doi.org/10.3989/arbor.2015.774n4003
Copyright: (c) 2015 CSIC. Este es un artículo de acceso abierto distribuido bajo los términos de la licencia Creative Commons Attribution-Non Commercial (by-nc) Spain 3.0.
RESUMEN: En el presente trabajo se estudia la vertiente poética de Francisco Umbral en el caso específico de la utilización de cláusulas de catorce sílabas, tanto en su escasa producción en verso como en su habitual escritura en prosa, incidiendo en el empleo de estos recursos en dos de las obras de la última época del escritor, los conocidos como Diarios íntimos, así como en el libro de poemas publicado póstumamente.

PALABRAS CLAVE: Alejandrino; Tetradecasílabo; Prosa poética; Métrica.
ABSTRACT: This work explores Francisco Umbral's poetic side, specifically through his use of fourteen syllables clauses, not only in his low production in verse but also in his usual prose writing, having persisted in using these techniques in two of the works from his final stage, known as Diarios intimos (Intimate Diaries), and also in the book of poems published posthumously.

KEYWORDS: Alexandrine; Tetradecasyllable; Poetic prose; Metric. 
"Contad si son catorce y está hecho"

El remate del conocidísimo soneto lopesco, aplicable al número de versos, me sirve para remontarme al cómputo de sílabas que supuso, en sus ya lejanos inicios, toda una revolución para las letras españolas ${ }^{1}$. El Mester de Clerecía inicia la nómina de variantes literarias hispanas que se verá incrementada, al paso de siglos y estilos, por nombres inscritos en el canon literario, por más que algunos sean de recientísima incorporación, como es el caso de Francisco Umbral, cuya impronta es ya, sin duda, estación de obligado descenso y estancia para los estudiosos.

Las características del alejandrino, sujetas en su mayor parte a irrenunciables rigideces formales, no parecen ser las más adecuadas a la hora de su estudio en un autor caracterizado por un uso del lenguaje libre, etéreo a veces, compacto otras, pero siempre dando la sensación de absoluta novedad estilística. Pero al mencionar esas catorce sílabas, con la hendidura de los dos hemistiquios heptasílabos, estamos hablando de un cómputo especialmente caro a un escritor no poeta; sí lirista no lírico; de espléndida prosa, de escaso estro (Couceiro, 2012, p. 305).

Este poema [...] Tiene poco valor lírico, sí, pero me sirve para psicoanalizarme literariamente [...] Lo primero que veo es mi reincidencia en el alejandrino, jugando a veces en hemistiquios (Umbral, 1996, p. 42).

Como punto de partida, estos conceptos requieren una puntualización: lirismo no es necesariamente lírica; cómputo silábico no significa ritmo sensu stricto; sensación estrófica no es precisamente musicalidad. Si lirismo corresponde al hecho de "aplicar características del estilo lírico en composiciones de otra clase" (DRAE, 22a ed.) frente a lírico, como vía que "trata de comunicar mediante el ritmo e imágenes los sentimientos o emociones íntimas del autor" (DRAE, 23a ed.); o si cómputo silábico se ajusta solamente a la métrica, al margen de secuencias internas, o, finalmente, si el concepto de musicalidad camina desde Grecia, incluso antes ${ }^{2}$, ensamblado con el Poema con mayúsculas, centrándome en este último concepto ¿cómo buscar musicalidad en un autor que, ab initio, afirma sus incapacidades musicales, tanto intrínsecas como de cultivo?: "Y no necesito decir, a estas alturas de la página, que yo detesto la música" (Umbral, 1996, p. 51). La explicación subsecuente al aserto no deja de ser alambicada, ya que el autor culpa al fenómeno musical del arrebatamiento, del éxtasis de su madre, con el alejamiento de esta de su hijo, quien se escuda en ello, revistiendo a la música de un grado alto de personificación que le permite sentirse celoso de ella. A este propósito, sería deseable un estudio amplio sobre la actitud de Umbral ante la música, que desprecia y respeta simultáneamente, en cierta concordancia con su actitud vital ante otras manifestaciones, porque a lo largo de su inmensa obra, raro es el libro en que no aparezca de uno u otro modo esta inquina. Lo cierto es que la música, para los no profesionales -y a veces, también para estos-, supone muchas veces un abandonarse a lo estético, con el añadido del hedonismo consecuente, que los atormentados no soportan ${ }^{3}$.

Como me aburre tanto la música, al joven cantante no le oigo, pero los violines son como cisnes hechos a mano, cisnes que suenan a madera y nunca tendrán la gracia fresca y tranquila de un lago, aunque toquen El lago de los cisnes. [...] Esta necesidad me ha llevado siempre a ser el aplaudidor ideal de los melodramas que no me gustan y de los conciertos que me van matando poco a poco. Un allegro más y me muero de tristeza (Umbral, 2001, pp. 31-32).

Umbral, poeta agónico, salpicado en detalles de amargura, unos, posiblemente intencionados; otros, camuflados en el lapsus linguae, pero todo ello motivo de evidente alerta para él como autor, como creador de un lenguaje a veces híbrido, a fuerza de estilismos perseguidos; Umbral, antimúsico confeso, como acabamos de ver, y sin embargo, sujeto al ritmo envolvente de la palabra, de la frase compacta, del período tenaz; Umbral, como Cervantes y Unamuno (Couceiro, 2012, p. 305), en busca de la poesía que intuye, que sabe, inalcanzable para él.

Escribe once años antes de su muerte:

Todo lo que he dado a la vida y a la literatura son los escombros de la frustración de un poeta lírico. A veces digo cínicamente [...] que escribo prosa porque la prosa se cobra, pero la verdad es que lo hubiera dado todo por escribir poesía, y a Dios le agradezco, al Dios alado de los poetas, que me abriese los ojos a tiempo (Umbral, 1996, p. 170).

Y tres años más tarde, ahonda en el mismo motivo, con algo de excusatio non petita:

Había nacido para poeta lírico y lo puse todo en prosa para vivir, para no ser un "lírico menesteroso", [...] yo descubrí enseguida que no quería hacer poesía pura, sino prosa impura (Umbral, 1999, p. 220).

Pues menos mal, porque a cambio, el autor arroja en una prosa deslumbrante el sucedáneo que más puede acercarse, eso que la crítica da en llamar "pro- 
sa lírica", y que no deja de remitir a la visión antirromántica de la que hablaba Mesonero Romanos, cuando escribía, al referirse al escritor de la época: "Rasguñó unas cuantas docenas de fragmentos en prosa poética y concluyó algunos cuentos en verso prosaico" (Mesonero Romanos, 1993, p. 301). Umbral conocía muy probablemente a Mesonero, y tal vez para curarse en salud, titula su primera edición de poemas de 1981 Antología de prosas líricas, en donde "prosas" y "líricas" están dando una clave de lectura que se sitúa decididamente en las antípodas del concepto cabal de poesía. Él lo sabe, y en este sentido, me parece pertinente lo que escribí en otra ocasión:

Un buen poema, un excelente verso no convierten en poeta a nadie y Francisco Umbral [...] es, en todo caso, un prosopoeta, un narratopoeta. A veces, incluso puede llegar a ser cronistopoeta, pero cuando intenta la poesía directa se queda [...] en el intento, en el esbozo, en la idea truncada (Couceiro, 2012, p. 306).

Hay que partir de la base de que la literatura, en su mayor parte, entra "por los ojos", sea desde la mancha, los blancos o las líneas versales. En nuestro autor llama la atención esa querencia por la prosa a nivel gráfico. Los mejores logros líricos de Umbral pertenecen a la mancha tipográfica discursiva, y la prueba del nueve es reconvertirlos en columnas versales, mientras que el caso contrario no funciona. He llevado a cabo el experimento con varios párrafos y poemas de distinta cronología umbraliana, e invariablemente, los resultados son similares. Basten dos expresivos ejemplos, el primero construido en alejandrinos, vector de este comentario:

1 a Original en prosa (mancha continua):

Ah tú, la vieja amante, cuánto cobre mentido en tu cuerpo desnudo, qué viejas cicatrices, como de cimitarra, te ha deparado el tiempo, tatuando tu hermosura. Ah tus ojos pequeños, tus pequeñas mentiras, tu pequeña vileza, que asoma entre tus dientes, ese sapo en tu cuello (Umbral, 2001, p. 182)

\section{1 b Desglose versal:}

Ah tú, la vieja amante, cuánto cobre mentido en tu cuerpo desnudo, qué viejas cicatrices, como de cimitarra, te ha deparado el tiempo, tatuando tu hermosura. Ah tus ojos pequeños, tus pequeñas mentiras, tu pequeña vileza, que asoma entre tus dientes, ese sapo en tu cuello
En el segundo caso se da la situación contraria desde el "verso", sin contenidos métricos:

\section{$\mathbf{2}$ a Original en líneas versales:}

Cuando noviembre, quizá diciembre, yerra por nuestra vida como un ángel custodio de marengo y de tos, la luz mayor, la luz luminosa, la luz que necesita uno para seguir viendo algo cuando ya no mira nada, resulta que no nos la da el sol ni el flash insistente, repetido, simultáneo, plural, de la actualidad en torno del que soy/no soy, sino que esa luz puede pasar momentánea, en el pelo de una muchacha, o vivir fija en el amarillo de una puerta. (Umbral, 2009, p. 116)

2 b Reelaboración en prosa:

Cuando noviembre, quizá diciembre, yerra por nuestra vida como un ángel custodio de marengo y de tos, la luz mayor, la luz luminosa, la luz que necesita uno para seguir viendo algo cuando ya no mira nada, resulta que no nos la da el sol ni el flash insistente, repetido, simultáneo, plural, de la actualidad en torno del que soy/no soy, sino que esa luz puede pasar momentánea, en el pelo de una muchacha, o vivir fija en el amarillo de una puerta.

Las diferencias saltan a la vista. La vista, ese es el punto de inflexión. Incluso si se hace la prueba de lectura en voz alta, la disposición discursiva del lector es completamente distinta ante la percepción métrica si la hay, como distinta es la actitud ante un final de línea, aunque tenga un guión de separación de palabra o un final de línea de verso. En el discurso umbraliano, es como si a niveles internos, el escritor se viese intimidado por la plasmación gráfica, como si las características de la página supusieran para él un hándicap bloqueador. Vemos que en $\mathbf{1}$ a, el discurrir continuo de la prosa en ningún momento evita la sensación métrica, no solo de las catorce sílabas, sino de la fuerte cesura del hemistiquio heptámetro, esté o no separado por comas. Eso nos permite la licencia de $\mathbf{1} \mathbf{b}$, que al trasladarse a líneas versales no pierde un ápice de calidad literaria; por el contrario, $\mathbf{2}$ a, a pesar de la evidente construcción en encabalgamientos, pierde belleza léxica y no gana ambiente lírico, por no mencionar la anarquía rítmica, mientras que al leerlo continuo, en $\mathbf{2} \mathbf{b}$, no solo se manifiesta un alto grado de lirismo sino que se realzan los valores literarios. De manera que, en el primer ejemplo ( $y$ hay centenares en Umbral) el ritmo fluye cómodo, proporcionado, y por eso es perfectamente verosímil convertirlo en líneas versales; en el segundo caso, al compactar en 
prosa el pseudopoema se recupera la "marca Umbral" sin fisuras, mientras que tal como él lo concibe, en líneas versales, no funciona, no hay ritmo ni cadencia aplicables al verso... porque es prosa. Resulta por eso sumamente interesante esa impronta referida a la mancha, puesto que el autor, cuya prosa es catalogada por tantos como lírica en alto grado, desbarra sistemáticamente cuando se trata de pretendidas columnas versales. Lo que en texto continuo le funciona, se estrella en cuanto lo convierte en línea versal. $Y$ no es que no lo intente, porque no faltan pruebas de ingenio de factura barroca, como cuando construye un poema al derecho para repetirlo como mancha tipográfica de prosa al revés, es decir, con las mismas frases pero en orden inverso y seguidas:

\section{(al derecho)}

El Rojito ha venido, ahilado de hospitales,

mi animal adorable ya orina en su rincón.

El Rojito ha venido como una hebra de gato,

como un gato magenta de las tintorerías. [...]

\section{(al revés)}

[...] Como un gato magenta de las tintorerías, el Rojito ha venido como una hebra de gato., mi animal adorable ya orina en su rincón, el Rojito ha venido, ahilado de hospitales (Umbral, 2008, pp. 86-87).

Hasta experimenta con un sistema disémico a lo largo de diez páginas, consistente en un solo verso por página, en una primera línea aislada, con el resto de la página en blanco. Así, ¿ंverso o viuda 4 ? porque Carta a mi mujer presupone un convencimiento del autor acerca de que España Suárez le sobreviviría, y Umbral, buen conocedor de los Barrocos, como acabo de señalar, conocía perfectamente estos alardes de agudeza que permiten polisemias semánticas (Díez Borque, 1993).

No sucede lo mismo con los recursos mecánicos. El autor, fuertemente enraizado en su/sus máquinas de escribir, a las que alude constantemente, a partir de aquella primera Olivetti, jamás se enfrentó al ordenador, y por tanto no pudo hacer ensayos con el juego propio de un programa de edición de textos que le hubiera permitido rápidamente variantes como el centrado, la variación de márgenes o los formatos experimentales:

La máquina me hizo prosista, aquella vieja Underwood [...]. Aquí se confirma una vez más la teoría vagamente marxista o materialista de que las condiciones exteriores, objetivas, determinan incluso el arte de un tiempo (Umbral, 1996, p. 136).
Cinco años después de este ejemplo, se reafirmará en la idea, en un capítulo completo de Un ser de lejanías (Umbral, 2001, pp. 60-61). El hábito hace al monje. $Y$ al servicio de esas premisas, al bucear en la ingente obra de este poeta deslucido, dos ritmos versales se destacan reiteradamente, más allá de épocas y estilos: la frase endecasílaba, la cláusula alejandrina. Esta, sobre todo. Y lo primero que llama la atención en alguien que se supone vanguardista y que, además, reniega de patrones -dice-, es la inmersión en una fórmula tan lejana en el tiempo, tan descaradamente medieval, como ya señalaba al inicio de este trabajo. Es cierto que los poetas de la Vanguardia rescataron, actualizándolos, el soneto como género y los cómputos tetradecasílabos como ritmo versal. Pues bien, es en este último caso donde el autor consigue, no solo notables hallazgos, sino frecuencia de uso, porque raro es el texto umbraliano donde no aparecen ejemplos de este recuento. Y no se trata tan solo del, aunque usual, caso aislado, dentro de un párrafo, sino del empleo sistemático del recurso al servicio de fragmentos enteros. En el caso de Umbral, uno de los lugares más apropiados para este procedimiento viene representado por la columna periodística o similares. Alrededor de setecientas / ochocientas palabras, lo que arrojaría una media de 110 a 130 cláusulas, cada una de ellas susceptible de convertirse en una línea versal. O aún mejor, algunos fragmentos de sus Diarios íntimos, en los que me voy a detener, dejando para mejor ocasión el mundo de la cuaderna vía periodística. Lo cierto es que el escritor empieza muy pronto a utilizar este recurso del alejandrino blanco continuo, pero según pasan los años, el sistema gana en eficacia y sensación de espontaneidad. Parece "salirle solo", como suele ocurrir cuando, tras dos horas de representación de una obra de Lope de Vega, muchos de los asistentes salen del teatro hablando en redondillas. El uso del alejandrino, dentro de la prosa, se hace más frecuente en la última década de vida del autor, coincidiendo con dos motivos. Por una parte, el incremento de su producción literaria en formato de Diario, que, a su vez, desemboca en el segundo motivo, que no es otro que la sucesiva mayor altura literaria de su discurso: "Un director de periódico, a mis cuarenta y tantos años, me dijo que yo estaba «muertecito». Pero en los veinte años siguientes el muertecito ha escrito sus mejores cosas" (Umbral, 2001, p. 198).

Así que en los siguientes párrafos me referiré a la franja de 1999 a $2001^{5}$, ya en los últimos años de Francisco Umbral, cuando sus "cantos del cisne" se hacen más explícitos: "Este largo libro es un mojón que pongo entre la muerte y yo", dice el 11 de noviembre de 1997 (Umbral, 1999, p. 86). 
Comenzando por el libro más intimista que inaugura su última década, el Diario político y sentimental, la frecuencia de la cláusula alejandrina se manifiesta en primer lugar, por el uso aislado, dentro del párrafo, pero cuya intención es focalizadora de contenidos densos o de vectores de sí mismo. En el último párrafo correspondiente al 29 de septiembre de 1997 (al comienzo del libro), reniega del pasado y del futuro para afirmar que "sólo somos presente" pero inmediatamente, ese aparente rechazo a lo que fue queda refutado con un único alejandrino: "y los viejos maestros a los que siempre vuelvo" (Umbral, 1999, p. 22). Este sistema de verso aislado lo emplea varias veces, muchas de ellas, precisamente en el último párrafo de la anotación diaria, y siempre es un contraste entre el contexto, como al describir algunos recuerdos materiales del día (personas, lugares), rompiendo la praxis del discurso con una pincelada poética, solo una: "hay un ángel de anís en el cielo festivo" (Umbral, 1999, p. 55). De nuevo en un último párrafo, tras la constatación de la gravedad de Carmen Díez de Rivera y el retrato descorazonador de su aspecto, es capaz de ensamblar un alejandrino con las mismas características, pero con el impacto que supone la fuerza rítmica del verso: "ojos de metal frío y un azul nada lírico" (Umbral, 1999, p. 69). Todavía con el mismo tema, y realzando la crueldad de la situación, dice también de Carmen: "amazona del mar, mujer de un solo pecho" (Umbral, 1999, p. 295), y seguimos encontrándolo en un último párrafo.

El 14 de diciembre de 1987 (de nuevo al final), hablando con cierto despego de la Transición en general y de Adolfo Suárez en particular, rompe el tono al decir que el expresidente genera aún "el respeto poético de un Doncel de Sigüenza" (Umbral, 1999, p. 88). Y siguiendo esta línea, el 21 de diciembre de 1997 el alejandrino no solo aparece en el último párrafo sino que lo cierra, en oposición a una "prosa" -el autor es explícito en este sentido-: "trenzada de las flores oscuras de diciembre"(Umbral, 1999, p. 143).

El texto correspondiente a una anotación diaria puede estar construido en cómputos versales en su totalidad. El viernes 3 de octubre de 1997 escribe este fragmento, en mancha continua, que, métricamente, resulta esclarecedor. Los versos los presento aislados, indicando el número de sílabas, destacando en negrita los de catorce. El protagonismo de los alejandrinos se hace evidente, pero debe señalarse el hecho de que hay tres endecasílabos ${ }^{6}$ y un pentadecasílabo que rompe el ritmo, como si quisiera con ello destacar ese aserto, ese "sí" incrustado para reafirmar el primer verso":
El sexo tiene días de cuchillo,

de violar a una virgen con un sable,

de beber las entrañas a una mujer morena, 14

de montar a una madre mientras reza a su hijo. 14

Hay días de cuchillo, una espada de sangre, 14

el falo es asesino, quiere dejar un rastro $\quad 14$

de mujeres tronzadas como muebles o muertos. 14

El sexo sí tiene días o noches de cuchillo, $\quad 15$

siempre el mejor amante es un criminal lento, 14

hay que brutalizar un desnudo de Ingres $\quad 14$

y lavarse las manos pulcramente $\quad 11$

o pedir un gin-tonic pronunciando bifiter. $\quad 14$

(Umbral, 1999, p. 30)

El viernes seis de marzo de 1998 escribe en el Diario uno de los párrafos más largos (dos páginas enteras), prácticamente completo en alejandrinos continuos, excepto dos o tres rupturas rítmicas. Se trata de un panegírico a Loyola de Palacio, ministra de Agricultura, Pesca y Alimentación en el primer Gobierno de José María Aznar (Cejudo García y Maroto Marcos, 1999). Pero ahora estamos ante otro trazado, porque en este fragmento la cuestión no es cuándo usa el alejandrino sino cuándo no lo usa.

Se inicia el capítulo con versos de alabanza que camina hacia la simbiosis mujer (Loyola) / producto de la naturaleza (aceite). Las características de la primera -virginidad, lucha- se entremezclan con el segundo -pureza, suavidad-. El vocativo, dos veces reiterado -"Ah"-, que no deja de parecer forzado en aras del cómputo silábico, es un recurso frecuente en este tipo de párrafos umbralianos:

Ah niña del aceite, Loyola del Olivo,

ah el puro aceite virgen y la virgen ministra,

se encontraron un día estas virginidades,

Eva blanca y adánica, clara serpiente verde,

y hoy Loyola se unge, se empeña en una lucha,

salvemos el aceite, salvemos los olivos,

sangre verde de España, herida y andaluza.

(Umbral, 1999, p. 217)

Sigue el siguiente párrafo con siete alejandrinos, desglosando alabanzas en construcción de zeugmas, y aparece la primera ruptura silábica al incrustar unos versos de Machado, seguidos de un breve comentario amétrico, para recuperar el cómputo tetradecasilábico en seis versos. Viene después otro párrafo más corto que mantiene el cómputo excepto en la última frase: 
Todas las cuentas cambian, también las de Bruselas,

pero ese viejo olivo, guerrero bajo el sol,

pero ese torso viejo, barroco y tan ibero,

debemos defenderlo, dejar que se defienda

debemos salvarlo de los hombres del agio. [13 sílabas]

(Umbral, 1999, p. 218)

En los dos últimos párrafos, solo rompe una vez el ritmo en cada uno, para terminar la anotación recogiendo la idea del inicio, incluidas las recurrencias al Paraíso terrenal y a la serpiente, con lo que el conjunto se cierra sobre sí mismo:

Avanza el bosque verde con filos de resoles,

el sol es arsenal de lancería,

[11 sílabas]

ni el socialista quiere ni mi Loyola quiere

que se lleven España arrancando los mapas.

Avanza el bosque verde, como el bosque de Shakespeare,

hacia el mal enemigo, hacia el negro contable

que les habla a las hojas con acento extranjero,

que morirá de bala de aceituna suavísima.

Loyola y la serpiente, estampario pagano,

Loyola y el aceite, rebelión de los campos.

Pan y aceite es España y una virgen violenta.

(Umbral, 1999, p. 219)

A propósito de este Diario político y sentimental, un último detalle: en la anotación correspondiente al 28 de diciembre de 1997, se encuentra, a mi juicio, el trozo más lírico de toda la obra, sin embargo, salvo dos o tres heptasílabos, todo lo demás es... en rigurosa prosa (Umbral, 1999, pp. 150-151).

Aunque él niega la mayor "esto que hago ahora no es una continuación del Diario" (Umbral, 2001, p. 93), el referente heideggeriano Un ser de lejanías es un evidente paralelo, solo que sin encabezamientos ni fechas marcadas en los parágrafos, siendo la única marca de corte el espacio en blanco de inicios y finales ${ }^{8}$. Se trata de un libro varias veces anunciado (incluso tres veces en un mismo día), concretamente, en la anotación del Diario que acabo de mencionar. El Umbral decadente, agonizante, moribundo, se intensifica en este otro canto del cisne, posiblemente su mejor prosa de los últimos años. Todos los ejemplos que siguen pertenecen a esta obra. Como en el caso precedente, los alejandrinos sueltos menudean:

me moriré en presente, con este viento alto (Umbral, 2001, p. 7)

-el destino no existe pero siempre es irónico-

(Umbral, 2001, p. 13) el espanto vacío de los días festivos (Umbral, 2001,

p. 114)

HAN venido a mi casa dos palomas de barro

(Umbral, 2001, p. 157)

TODO lo que yo toco tiene un resol de muerte (Umbral, 2001, p. 192)

posteridad anónima de mi vencido cuerpo (Umbral, 2001, p. 206)

Qué gran rey solitario, anterior a los griegos (Umbral, 2001, p. 172)

Qué tranquilo en lo eterno, qué fugaces los hombres (Umbral, 2001, p. 172).

También pueden ir consecutivos, de dos en $\operatorname{dos}^{9}$ :

EL velamen de octubre me golpea en el pecho.

Una ciudad nublada se rehace a la deriva.

(Umbral, 2001, p. 16)

Hay un joven judío, competidor y amable,

que me recuerda textos de "antijudaísmo".

(Umbral, 2001, p. 151)

O de tres en tres:

yace la mariposa de mi mirada miope, descompuesta en colores, metáforas o fórmulas.

La verdad de las cosas, tan sencilla y sangrienta.

(Umbral, 2001, p. 13)

A veces, los alejandrinos son un anclaje para el texto que sigue, y luego se van abandonando; arrancan con vigor, para perderse a los tres o cuatro versos y recuperarse al final. $\mathrm{O}$ bien, caminan entretejidos por entre la prosa del fragmento:

HOJA de parra, otoño, candela de domingo,

mi luz mientras escribo, hoja de bronce pálido [...]

Afuera está el caudal de un domingo que pasa [...]

Salgo a respirar día, invierno venidero [...]

Ah miles de domingos, cuchillo del recuerdo.

(Umbral, 2001, p. 26)

Luego vienen, claro, los capítulos enteros -o casi-, en los que si se rompe el ritmo una o dos veces, se percibe la intención de esa ruptura como realce del contenido, como sucede en "SU voz en el teléfono". $\mathrm{O}$ a medida que el contenido se endurece de quejas, rencores, resentimientos; o en los fragmentos anafóricos cuyo motivo reiterado es "Lanza tu coche rojo":

LANZA tu coche rojo y estréllalo en el viento, muramos en la llama verde de los crepúsculos, 
llévame hasta el final clamoroso del día

y nuestras dobles vidas, nuestras dos juventudes, que reflorezcan lejos de nuestra biografía. [...]

Lanza tu coche rojo y estréllalo en el tiempo [...]

acabemos de golpe con la farsa brillante

de estar vivos por siempre en los saraos de sangre.

(Umbral, 2001, p. 155)

O en "Ah, tú, la vieja amante":

AH TÚ, la vieja amante, hoy dispersa en culebras, el tiempo ha hecho una máscara sobre tu rostro ofidio, cae una sombra mala sobre tu risa falsa, reinas en las palabras como en tu gusanera, las palabras que salen de tu boca no llegan [...] eres un gran silencio que profiere lenguajes.

Ah tú, la vieja amante, cuánto cobre mentido en tu cuerpo desnudo, qué viejas cicatrices, como de cimitarra, te ha deparado el tiempo, tatuando tu hermosura. Ah tus ojos pequeños, tus pequeñas mentiras, tu pequeña vileza, que asoma entre tus dientes, ese sapo en tu cuello, [...]

Ah la maldad estúpida, la que no vale nada, [...] y el tiempo no te ha dado grandeza ni destino, [...] Te depuran los años, afilan tus puñales, pero nada has cambiado, mujer de boca dura.

(Umbral, 2001, p. 182)

Y el fragmento termina con una serie de frases fuera de ritmo, solo interrumpidas con un único verso: "toda la vieja sangre bordada entre tus pechos" (Umbral, 2001, 182). Este motivo de la sangre se reiterará en varias zonas del libro:

LA interrumpida sangre, la sangre de mi sangre [...]

la sangre de la herida, la sangre gratuita con su infantil violencia, cervatillo encarnado que brinca por mi vida, gracia fluvial, violenta, de la observada sangre, de la olvidada sangre, la solitaria sangre que viaja mi cerebro [...] alambrada de sangre que protege mi vida.

(Umbral, 2001, pp. 117-118)
Porque el concepto de anatomía decadente es constante a lo largo de todo el texto, reforzando el concepto-vector de "Ser de lejanías":

Ah el amor cadavérico del adulterio póstumo, sonantes esqueletos que aún dan alegre música, así pasan mis tardes, nuestras fugaces tardes [...] (rompe el ritmo y sigue)

la veo cómo se aleja, desnuda entre la gente, con la cabeza baja, morenía que amo,

y su revés esbelto, ah sus fluyentes piernas, vibrante todavía de los últimos besos, pero otra vez tristísima, falsa niña asustada, ante la inmensa sombra que la ciudad proyecta, sombra que hacia ella avanza, ademán del destino (rompe el ritmo y sigue)

en amores perdidos, deshojada en palabras.

(Umbral, 2001, p. 141)

Un último caso, el capítulo completo en alejandrinos, solo sesgado por tres brevísimas interrupciones:

ESE tropel de carne que pasa por tu cuerpo (rompe el ritmo y sigue)

primavera del sexo, violencia de tu edad, qué juventud perfuma tu profunda vagina.

Tu cabeza francesa, tu mirada sonríe (rompe el ritmo y sigue)

ah niña de Versalles celebrando los cuerpos. Pisando peñascales, ah la Fuente del Berro, he llegado a tu lecho, barroco, almidonado que se deshace tenue, ya como un cuerpo él mismo.

(rompe el ritmo y sigue hasta el final...)

Por qué viniste entonces y regresas ahora, por qué la edad me trajo, como una fiesta a solas, tu desnudo de sangre, la avidez de tu boca.

Mas no es tu religión lo que canta este libro, sino la hora penúltima, campanas de tu barrio, en que fornico y canto como un general ebrio, sabiendo ya muy cerca el tiempo de los muertos, la otoñada que espera en mi alto cementerio. Por qué la vida, digo, tan cerca de la muerte, y un puñado de rosas, tomado de tu cuerpo, sonando en los maitines de mi cansada vida. Estás lejos ahora, tu voz entre los mares, 
pero eso ya no vale, la vuelta como farsa, quiero decir tan sólo, mientras sangro despacio, que fuiste el ángel malo, el dios hembra de vino que cultivó mi vida cuando ya sólo un dólar

(deja el verso final en la mitad, con un heptasílabo) me quedaba en el pecho.

(Umbral, 2001, pp. 149-150)

No puedo terminar este trabajo sin detenerme brevemente en los versos de Umbral sensu stricto, lo que él (ya lo señalé) denominaba "prosas líricas" en 1981, y que su editor Miguel García Posada titula Obra poética (1981-2001). Tras lo que antecede, esta publicación póstuma es quizá la prueba más evidente de que Umbral como poeta baja muchos peldaños en comparación a la luminosidad de su prosa, por más que esta claudique al ritmo y a la métrica con la frecuencia que acabamos de ver. Sin embargo, lo que puede salvar el libro son, precisamente, esa especie de poemas en prosa, muy abundantes, que presentan dos modelos: la mancha continua -más o menos- o la línea versal.

En el primer caso, hay una diferencia con los Diarios consistente en que los fragmentos no métricos son alineados a la izquierda, algo familiar para quienes utilizan ordenador, pero aún más para las viejas máquinas de escribir, que no tienen la posibilidad de la mancha justificada. La mayoría de estos casos es pura prosa, pura y bellísima prosa, pero prosa. Hasta tal punto, que hay nueve "apartados", sin título, que cabrían perfectamente insertados en su Mortal y rosa. El mismo estilo, el mismo contenido. Incluyo el último, brevísimo, respetando el formato:

Sólo he vivido cinco años de mi vida. Los cinco años

que vivió mi hijo. Antes y después, todo ha sido caos y crueldad (Umbral, 2009, p. 63).

Sin embargo, a la hora de utilizar el cómputo correspondiente al alejandrino, Umbral ofrece dos variantes: la línea a línea, manteniendo las catorce sílabas en cada verso:

Pega, muchacha, pega, con tu melena látigo, azota la tristeza sobre mis atrios fríos,

despójame de historia, repite el vuelo lento de tu melena negra, pájaro tan tupido, que como un ala sola, muy cargada de sexo, pasa sobre los cuerpos cadáver de domingo. (Umbral, 2009, p. 34)
$U$ otra variante consistente en el alejandrino continuo (que hemos visto en los dos libros ya comentados), pero en estos la mancha estaba justificada, y en la Obra poética aparecen, como acabo de mencionar para las prosas, alineados a la izquierda, con lo que se da la paradoja de líneas versales arbitrarias al servicio de alejandrinos rigurosos, que pueden ser blancos:

\begin{abstract}
Hoy que la lluvia me abre lúgubre sus salones, cuando el silencio sube negro hasta las palabras, hoy que la muerte toma forma casi apacible, quiero evocar con humo, como se le habla al fuego, esa voz tan dolida, esos perennes ojos (Umbral, 2009, p. 133).
\end{abstract}

O con rima asonante:

Calle de tantos astros, rinconada del tiempo, la dimensión del mundo me la daba un vencejo. Oro de las mañanas empobreciendo el cielo, soles de cada tarde en un ladrillo eterno, de los países del alba venían los buhoneros y en sus pregones altos flotaba un hombre muerto (Umbral, 2009, p. 66).

Hay un total de veinte composiciones alejandrinas en la primera parte del libro, Crímenes y baladas (1981), mientras que en la segunda parte, Poemas (2000-2001), al margen de algunos escasos tetradecasílabos salpicados en poemas, mayoritariamente de Arte menor, solo hay un caso aislado, completamente alejandrino (aunque tiene dos trozos en que los desdobla a heptasílabos), el poema La muerte, a lo que habría que añadir otro poema completamente en heptasílabos (Bardem) que, naturalmente, remite a hemistiquios seguidos, creando un ritmo paralelo. En este sentido, llama la atención el desequilibrio entre la primera parte, con tantos ejemplos de métrica tetradecasilábica, coincidente cronológicamente (los ochenta) con una producción del autor centrada en una alta cifra de novelas, en las que no abunda el recurso, mientras que la segunda parte (2000-2001), apenas sin ejemplos de este metro, camina afín en escritura con los Diarios que acabamos de ver, y que tan pródigamente emplean la cláusula alejandrina.

El análisis en segundo grado de cada uno de los fragmentos versales de estas características en la Obra Poética requeriría una investigación aparte, y eso me lleva a considerar el orden del epígrafe que inicia estas líneas con el número (I). Teniendo en cuenta que, como también señalé 
al principio, está pendiente un estudio del alejandrino en la vertiente columnística del autor, no parece aventurado adelantar que este tipo de buceo va a necesitar más de un trabajo añadido.

Lo que parece evidente, tanto por las declaraciones del propio Umbral como por la frecuencia de su uso, es la importancia que supone el juego de los alejandrinos dentro de su obra, sobre todo en aquella más cercana al intimismo, que no por ello va de espaldas a su capacidad de lector ávido, de crítico, de teórico. En definitiva, "de forma implícita, en muchas de sus páginas, vuelca sus teorías literarias [...] Umbral tenía las ideas muy claras" (Rodríguez Pequeño, 2012, p. 98). Un punto más a favor de un autor mucho más respetuoso con la forma, el plano o la estructura de lo que podría parecer a sus lectores, si estos se quedan en una primera visión receptiva. Cierro pues estas páginas en abierto, con la propias palabras de Francisco Umbral, a propósito de una de sus frecuentes declaraciones de principios:

Si mi pensamiento tiene capacidad de borrar un dolor, también la tiene de crear una figura y una escritura. Y duermo dejando a un lado, muy cerca, mi pensamiento y mi fuerza, como el clásico dejaba su Biblia y su espada (Umbral, 2001, p. 41).

\section{NOTAS}

[1] La Cuaderna vía, o estrofa de cuatro versos monorrimos, de catorce sílabas, divididos en dos hemistiquios de siete, fue el mayor cauce para la poesía culta española durante los siglos XIII y XIV. Recibe su nombre de la descripción que aparece en la segunda estrofa del anónimo Libro de Aleixandre

Mester traigo fermoso, non es de joglaría

mester es sin pecado, ca es de clerezía,

fablar curso rimado por la quaderna vía

a sílabas contadas, ca es grant maestría.

[2] Los testimonios poéticos ya están en Egipto (Textos de la Pirámides $\mathrm{V}$ y VI Dinastías); en Sumeria (Poema de Gilgamesh, s.VII a. C.); o Israel (Textos poéticos veterotestamentarios -Los Salmos o El Cantar de los Cantares-), anteriores o coetáneos a Grecia.

\section{BIBLIOGRAFÍA}

Libro de Alexandre (1995) (ed. J. Cañas). Madrid: Cátedra.

Cejudo García, E. y Maroto Marcos, J. (1999). Pasado, presente y futuro de la OMC del aceite de oliva. Cuadernos Geográficos, 29, pp. 85-117.

Couceiro, M. P. (2012). Prosopaemos. En: Díez, J. I. (ed.). Los placeres literarios. Francisco Umbral como lector. Madrid: Fundación Francisco Umbral, pp. 305314.

Couceiro, M. P. (2013). Textos bíblicos e implicaciones musicales en el teatro bajomedieval y renacentista. Biblias Hispánicas, 2, pp. 245-258.
[3] Este concepto musical de "abandono" está secularmente presente en áreas eclesiásticas, y para explicar algunas razones del alejamiento general de muchos españoles hacia la cultura musical, habría que remontarse a Decretos tridentinos, que durante siglos, y a niveles sociales, han atravesado la columna vertebral de la historia de España.

[4] En tipografía, una línea viuda es aquella que, siendo la última de un párrafo, aparece al principio de la página o columna siguiente, por lo que se muestra aislada de su contexto y, por norma general, seguida de una línea o varias en blanco que la separan del siguiente párrafo.

[5] La Obra Poética es publicación póstuma, en 2009, pero los poemas recogidos en ella llegan hasta 2001.

Díez Borque, J. M. (1993). Verso e imagen Del Barroco al Siglo de las luces. Madrid: Calcografía Nacional y Dirección General del Patrimonio Cultural de la Comunidad de Madrid.

Diccionario de la Real Academia Española (vigésima segunda edición).

Diccionario de la Real Academia Española (avance de la vigésima tercera edición).

Mesonero Romanos, R. de (1993). Escenas y tipos matritenses (ed. E. Rubio Cremades). Madrid: Cátedra.

Rodríguez Pequeño, M. (2012). Francisco Umbral, historiador, teórico y crítico literario. En: Díez, J. (ed.). Los placeres li-
[6] Umbral también se vale a veces del endecasílabo para largos procesos, pero, frente a las catorce sílabas, las once se quedan en un mínimo porcentaje.

[7] A partir de este momento, todos los ejemplos originales en "prosa continua" los ofrezco desglosados en versos.

[8] Algunos fragmentos del Diario político y sentimental no estaban fechados pero eran fácilmente ubicables al estar entre otros que sí tenían el dato cronológico definido.

[9] Como en el caso anterior, escribo los alejandrinos en líneas distintas, aunque en el original estén continuos.

terarios. Francisco Umbral como lector. Madrid: Fundación Francisco Umbral, pp. 97-114.

Umbral, F. (1996). Los cuadernos de Luis Vives. Barcelona: Planeta.

Umbral, F. (1999). Diario político y sentimental. Madrid: Círculo de Lectores.

Umbral, F. (2001). Un ser de lejanías. Barcelona: Planeta.

Umbral, F. (2008). Carta a mi mujer. Barcelona: Planeta.

Umbral, F. (2009). Obra poética (19812001) (ed. M. García Posada). Barcelona: Seix Barral. 